\title{
Detection method of space-time conflict for multi route planning
}

\author{
Chong $\mathrm{Li}^{1}$, and Yanyan Huang ${ }^{1, *}$ \\ ${ }^{1}$ School of Automation, Nanjing University of Science \& Technology, Nanjing, China
}

\begin{abstract}
In order to solve the problem of space-time conflict in multi route planning, this paper proposes a space-time conflict detection method based on bounding box. The main research contents include: dividing each route into a limited number of road sections, each road section uses a bounding box to represent the space occupied by the target in the period of time, and then using OBB collision detection method to judge whether there is different bounding box overlap in the same space at the same period of time, so as to judge whether there is a space-time conflict. In addition, calculating the distance among road sections to exclude road sections where conflicts are unlikely to occur before detection. The feasibility of the conflict detection method is verified by experiments on a multi route planning case.
\end{abstract}

\section{Introduction}

Before an aircraft performing a mission, formulating a flight route plan for the aircraft is necessary. The flight route plan includes time, position of the aircraft. When formulating the route plan with a number of aircrafts, it is necessary to consider whether there is a possible conflict among those aircrafts in the flight process to ensure the safety of the aircrafts. When formulating a route plan, we should avoid different aircrafts being in the same position at the same time. This is the problem of space-time conflict when formulating the multi route plan.

In order to solve the problem, it is necessary to detect the space-time conflict to judge which aircraft in the flight route plan may collide at what time and at what position. Then the operator who formulate the flight plan can know how to adjust the plan.

Many scholars have carried out research on space-time conflict detection ${ }^{[1-2]}$. Most research focuses on the space-time conflict when executing the plan ${ }^{[3-4]}$ or space conflict without time ${ }^{[5]}$, but little research on space-time conflict when formulating the plan is studied.

At present, space-time conflict detection method is still not mature. So, A new space-time conflict detection method for multi route planning is presented in this paper.

\section{Space-time conflict detection for multi route planning}

\subsection{The expression of time and space in space-time conflict analysis}

To consider the problem of space-time conflict, we should determine the expression of time

*Corresponding author: imhyy@sina.com 
and space at first. In this paper, we consider using the period of time to represent time, and using the bounding box occupied by each period of time to represent space.

Giving the route $L$ in three-dimensional space, $L$ contains many waypoints, the information of waypoint includes the aircraft's position and time, so $L$ can be represented by a Two-Tuple $\langle P, T\rangle$, Variable set $P=\left\{p_{1}, p_{2}, \ldots, p_{n}\right\}$ represent all positions of the route $L$, Variable set $T=\left\{t_{1}, t_{2}, \ldots, t_{n}\right\}$ represent all time of the set of the position $P$.

For a given period of time $\left[t_{s}, t_{e}\right]$, if $t_{s}$ is greater than the start time of route $L, t_{e}$ is less than the end time of route $L$, and $t_{s}$ is less than $t_{e}$, assuming that the aircraft is traveling in a straight line between two adjacent waypoints, then the route within the period of time $\left[t_{s}, t_{e}\right]$ can be uniquely determined, and the position of the aircraft at $t_{s}$ is:

$$
\left\{\begin{array}{c}
p_{s}=\left(x_{s}, y_{s}, z_{s}\right) \\
x_{s}=x_{i}+\left(t_{s}-t_{i}\right) *\left(x_{i+1}-x_{i}\right) /\left(t_{i+1}-t_{i}\right) \\
y_{s}=y_{i}+\left(t_{s}-t_{i}\right) *\left(y_{i+1}-y_{i}\right) /\left(t_{i+1}-t_{i}\right) \\
z_{s}=z_{i}+\left(t_{s}-t_{i}\right) *\left(z_{i+1}-z_{i}\right) /\left(t_{i+1}-t_{i}\right) \\
t_{s} \geq t_{i} \\
t_{s} \leq t_{i+1}
\end{array}\right.
$$

In this formula, $x_{i}$ represents the $\mathrm{x}$ - axis coordinate of the $\mathrm{i}$-th waypoint, $x_{i+1}$ represents the $\mathrm{x}$-axis coordinate of the $\mathrm{i}+1$-th waypoint, $y_{i}$ represents the y-axis coordinate of the i-th waypoint, $y_{i+1}$ represents the y-axis coordinate of the i+1-th waypoint, and $z_{i}$ represents the $\mathrm{z}$-axis coordinate of the $\mathrm{i}$-th waypoint, $z_{i+1}$ represents the $\mathrm{z}$-axis coordinate of the $\mathrm{i}+1$-th waypoint, $t_{i}$ represents the time of the $\mathrm{i}$-th waypoint, and $t_{i+1}$ represents the time of the $\mathrm{i}+1$-th waypoint.

In the same way, the position of the aircraft at $t_{e}$ can be derived. The space occupied by objects in the period of time $\left[t_{s}, t_{e}\right]$ can be represented several bounding boxes.

The bounding box belonging to a road section is represented a cuboid, as the figure 1 shows. In this figure, $\mathrm{P} 1$ and $\mathrm{P} 2$ are two adjacent waypoints. The connection between the two waypoints constitutes a road section P1P2. A1B1C1D1A2B2C2D2 is the bounding box of the road section P1P2. O1 and $\mathrm{O} 2$ are the centers of the squares A1B1C1D1 and A2B2C2D2 respectively. A1B1C1D1 and $\mathrm{A} 2 \mathrm{~B} 2 \mathrm{C} 2 \mathrm{D} 2$ have side lengths of $2 * d_{\text {safe }}$, where $d_{\text {safe }}$ is safe distance. A1A2, B1B2, C1C2, D1D2 are parallel to P1P2, A1B1C1D1 and A2B2C2D2 are perpendicular to $\mathrm{P} 1 \mathrm{P} 2$.

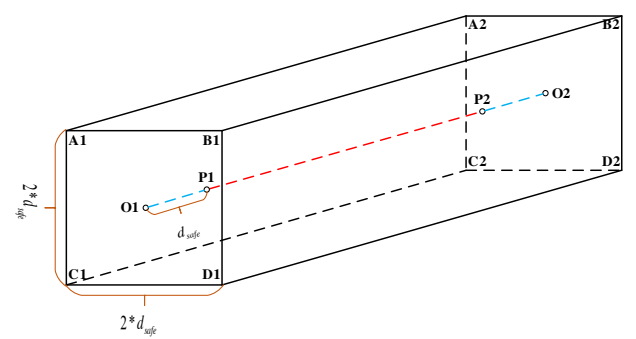

Fig. 1. Bounding box diagram.

The position of each vertex of the bounding box of any road section can be determined 
according to the safe distance $d_{\text {safe }}$ and the positions of two waypoints of the road section, so the space occupied in the period of time by the aircraft can be uniquely determined.

From the above, giving any period of time, the space occupied by the aircraft can be determined in that period of time. So, the flight time can be divided into finite periods of time, and each period of time has a space which the aircraft occupied in that period of time.

\subsection{Space-time conflict detection}

By analysis, time is represented by period of time, space is represented by bounding box. So, to judge whether a space-time conflict occurs, we only need to judge whether the space occupied by different aircraft in the same period of time overlap.

\subsubsection{Space-time conflict detection based on OBB}

The OBB (Oriented Bounding Box) detection method was proposed by Gottschalk in 1988, which is used for conflict detection of oriented bounding boxes. Since a cuboid is used to represent the space, and the orientation of the cuboid is uncertain, the cuboid can be regarded as an oriented bounding box, and the OBB conflict detection method is used to judge whether there is different bounding box overlap. Generally, the separation axis theorem and the separation axis theorem are used: if an axis can be found that the projections of two convex objects on this axis do not overlap, then the two objects do not intersect, if an axis cannot be found that the projections of two convex objects on this axis do not overlap, then it can be determined that the two objects intersect. For OBB conflict detection of two cuboids, you only need to find a separating axis to determine whether the two cuboids intersect. There are 15 candidate separation axes: 3 coordinate axes of one cuboid, 3 coordinate axes of the other cuboid, and 9 axes perpendicular to a certain axis. You only need to judge whether the projections on these 15 axes overlap to judge whether the two objects intersect.

\subsubsection{Optimization of Space-time conflict detection based on distance judgment}

OBB conflict detection is very time-consuming, so it is necessary to minimize the times of using OBB conflict detection to improve the method performance. By analysis, OBB conflict detection is not necessary in the following case. When the distance between the two bounding boxes is too far, that is, the distance between the centers of the two bounding boxes is greater than the sum of the radii of the circumscribed sphere of the two bounding boxes, then there is no need to use OBB conflict detection.

We can easily calculate the radii of the circumscribed sphere of the bounding box and the distance between the centers of the two bounding boxes, so wo just make sure:

$$
\left\{\begin{array}{c}
d_{c}>\varepsilon \\
d_{c}=\left(\left(x_{c 1}-x_{c 2}\right)^{2}+\left(y_{c 1}-y_{c 2}\right)^{2}+\left(z_{c 1}-z_{c 2}\right)^{2}\right)^{1 / 2} \\
\varepsilon=\left(\left(d_{\text {safe }}+d_{1} / 2\right)^{2}+\left(\sqrt{2} * d_{\text {safe }}\right)^{2}\right)^{1 / 2}+\left(\left(d_{\text {safe }}+d_{2} / 2\right)^{2}+\left(\sqrt{2} * d_{\text {safe }}\right)^{2}\right)^{1 / 2}
\end{array}\right.
$$

In this way, there is no need to use OBB conflict detection, among them, $\varepsilon$ is the sum of the radii of the circumscribed sphere of the two bounding boxes, and $d_{c}$ is the distance between the centers of the two bounding boxes. 


\subsection{Algorithm flow}

The algorithm flow is shown in figure 2. In this figure, $L=\left\{L_{1}, L_{2}, \ldots, L_{n}\right\}$ is the set of routes, $r_{i k}$ is the k-th road section in the i-th route. $p t_{i k}$ is the period of time of $r_{i k}, C_{i k}$ is the bounding box of $r_{i k}, d_{c_{-} i k j m}$ is the distance between the center of $r_{i k}$ and the center of $r_{j m} . \varepsilon_{i k j m}$ is the sum of the diameters of the circumscribed sphere of $C_{i k}$ and $C_{j m}$. $\operatorname{len}\left(L_{i}\right)$ is the number of road section in $L_{i} . O B B\left(C_{i k}, C_{j m}\right)$ means using OBB detection method to judge whether there is a conflict between $C_{i k}$ and $C_{j m}$.

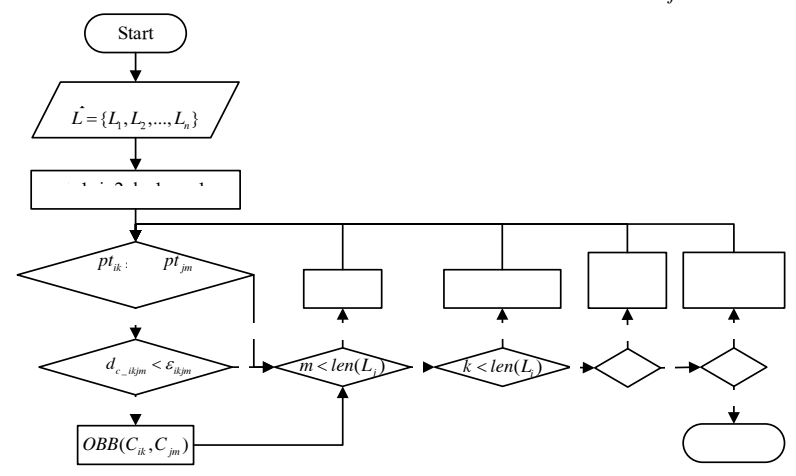

Fig. 2. Algorithm flowchart.

\section{Case analysis}

Two routes in a given Cartesian coordinate system:

$L_{1}=\{(10,10,0,10: 00),(35,35,20,10: 10),(67,70,45,10: 20),(100,90,70,10: 30)$,

$(122,118,100,10: 40),(162,143,100,10: 50)\}$

$L_{2}=\{(18,202,0,10: 15),(69,191,20,10: 25),(110,173,40,10: 35),(137,141,100,10: 45)$,

$(177,116,100,10: 55),(219,96,100,11: 05)\}$

If a road section is formed between two adjacent waypoints, $L_{1}$ includes 5 sections:

$$
\begin{gathered}
r_{11}=\{(10,10,0,10: 00),(35,35,20,10: 10)\} \\
r_{12}=\{(35,35,20,10: 10),(65,70,45,10: 20)\} \\
r_{13}=\{(65,70,45,10: 20),(100,90,70,10: 30)\} \\
r_{14}=\{(100,90,70,10: 30),(122,118,100,10: 40)\} \\
r_{15}=\{(122,118,100,10: 40),(162,143,100,10: 50)\}
\end{gathered}
$$

the route $L_{1}$ includes 5 sections:

$$
\begin{gathered}
r_{21}=\{(18,202,0,10: 16),(69,191,20,10: 26)\} \\
r_{22}=\{(69,191,20,10: 26),(110,173,40,10: 36)\} \\
r_{23}=\{(110,173,40,10: 36),(137,141,100,10: 46)\} \\
r_{24}=\{(137,141,100,10: 46),(177,116,100,10: 56)\} \\
r_{25}=\{(177,116,100,10: 56),(219,96,100,11: 06)\}
\end{gathered}
$$

Firstly, judging whether the period of time of route L1 and route L2 overlap, and finding there are 7 groups of overlapped road section combinations: $g_{1}=\left(r_{12}, r_{21}\right), g_{2}=\left(r_{13}, r_{21}\right)$, 
$g_{3}=\left(r_{13}, r_{22}\right), g_{4}=\left(r_{14}, r_{22}\right), g_{5}=\left(r_{14}, r_{23}\right), g_{6}=\left(r_{15}, r_{23}\right), g_{7}=\left(r_{15}, r_{24}\right)$. Let $d_{\text {safe }}=4$.

Calculating the sum of the radii of the circumscribed sphere of the two bounding boxes of each road section combination named $\varepsilon$ and the distance between the centers of the two bounding boxes of each road section combination named $d_{c}$ by formula (2). Then you can get $\varepsilon_{1}=63.1, \varepsilon_{2}=60.7, \varepsilon_{3}=58.3, \varepsilon_{4}=57.8, \varepsilon_{5}=68.7, \varepsilon_{6}=69.1, \varepsilon_{7}=56.3$, $d_{c 1}=145.9, d_{c 2}=131.7, d_{c 3}=105.9, d_{c 4}=97.7, d_{c 5}=56.5, d_{c 6}=32.0, d_{c 7}=15.1$. So, $d_{c 1}>\varepsilon_{1}, d_{c 2}>\varepsilon_{2}, d_{c 3}>\varepsilon_{3}, d_{c 4}>\varepsilon_{4}, d_{c 5}<\varepsilon_{5}, d_{c 6}<\varepsilon_{6}, d_{c 7}<\varepsilon_{7}$. According to the formula (2), it can be seen that there may be conflicts in the road section combinations $g_{5}=\left(r_{14}, r_{23}\right), g_{6}=\left(r_{15}, r_{23}\right), g_{7}=\left(r_{15}, r_{24}\right)$. Constructing bounding boxes $C_{14}, C_{15}, C_{23}$, $C_{24}$ of road sections $r_{14}, r_{15}, r_{23}, r_{24}$ respectively. Performing OBB conflict detection on $C_{14}$ and $C_{23}, C_{15}$ and $C_{24}, C_{15}$ and $C_{23}$ respectively, and get the conclusion that there is a conflict between $C_{15}$ and $C_{24}$, that is, there will be a space-time conflict between the road section $r_{15}$ in $L_{1}$ and the route section $r_{24}$ in $L_{2}$.

To verify the result, assuming that the aircraft is traveling in a straight line at a constant speed in the road section, when $\mathrm{t}=10: 47: 30$, the waypoint position on route $L_{1}$ at this time can be calculated to be $(152,136,75)$, and the waypoint position on route $L_{2}$ at this time can be calculated to be $(155,134,75)$, and the distance between the two waypoints at this time is calculated to be 3.6, which is less than the safe distance. So, there is a risk of conflict, that is, a space-time conflict will occur.

\section{Conclusion}

This paper studies the space-time conflict detection in multi route planning. In the past, most research on the space-time conflict detection is about the flight process. The time in the flight route planning in this paper is regarded as a set of periods of time, and space is regarded as a bounding box occupied by each aircraft in different periods of time. By judging whether there is different bounding box overlap in the same space at the same period of time, it can be derived that whether there is a time-space conflict in flight route plan. Finally, the effectiveness of the method is verified by experiments. However, the method has certain requirements for the interval of periods of time, if the period of time is too long, the method may judge a route that will not conflict actually as a route that will conflict.

\section{References}

1. S. Hao, S. Cheng, Y. Zhang, A multi-aircraft conflict detection and resolution method for 4-dimensional trajectory-based operation, Chinese Journal of Aeronautics, 31, 15791593 (2018)

2. M. Sun, K. Rand, C. Fleming, 4 Dimensional waypoint generation for conflict-free trajectory based operation, Aerospace Science and Technology, 88 (2019)

3. J. Kuchar, L. Yang, A Review of Conflict Detection and Resolution Modeling Methods, IEEE Transactions on Intelligent Transportation systems, 1, 179-189 (2000)

4. P. Hubbard, Collision detection for Interactive Graphics Applications. IEEE Transactions on Visualization and Computer Graphics, 1, 218-230 (1995)

5. E. Arbabi, R. Roulic, D. Thalman. Fast Collision Detection Methods for Joint Surfaces. Journal of Biomechanics, 42, 91-99 (2009) 